\title{
Geotechnical Investigation and Prediction of Rock Burst, Squeezing with Remediation Design by Numerical Analyses along Headrace Tunnel in Swat Valley, Khyber Pakhtunkhwa, Pakistan
}

\author{
Mian Sohail Akram*, Kamran Mirza, Muhammad Zeeshan, Muhammad Ali, Luqman Ahmed \\ Institute of Geology, University of the Punjab, Lahore, Pakistan \\ Email: *sohail.geo@pu.edu.pk, Kamran.geo@pu.edu.pk, muhammadzeeshanpak@gmail.com, ali.geologist001@gmail.com, \\ sh_luqman@yahoo.com
}

How to cite this paper: Akram, M.S. Mirza, K., Zeeshan, M., Ali, M. and Ahmed, L. (2018) Geotechnical Investigation and Prediction of Rock Burst, Squeezing with Remediation Design by Numerical Analyses along Headrace Tunnel in Swat Valley, Khyber Pakhtunkhwa, Pakistan. Open Journal of Geology, 8, 965-986. https://doi.org/10.4236/ojg.2018.810058

Received: August 12, 2018

Accepted: September 18, 2018

Published: September 21, 2018

Copyright (c) 2018 by authors and Scientific Research Publishing Inc. This work is licensed under the Creative Commons Attribution International License (CC BY 4.0).

http://creativecommons.org/licenses/by/4.0/

\begin{abstract}
This study illustrates the classification of the rock mass and evaluation of rock squeezing, rock burst potential, deformation modulus along the proposed tunnel alignment of small hydropower in Swat Valley, Khyber Pakhtunkhwa (KP), Pakistan. The field and laboratory studies were conducted to classify the rock mass by using geomechanical classification systems i.e. Rock Mass Rating (RMR), tunneling quality index (Q), Rock Mass Index (RMi). The empirical relations classified the ground as non-squeezing and minor to non-squeezing conditions, respectively. Whereas, other methods depict minor to medium bursting potential along chainage $1+000$ to $4+000 \mathrm{~m}$, while results along chainage $2+400-2+800 \mathrm{~m}$ present medium to high bursting potential. Furthermore, numerical analyses were carried out by $\mathrm{RS}^{3}$ for elastic and plastic conditions in order to assess the total displacement of each section in unsupported and supported conditions. The results gave maximum displacement along chainage 2+400 - 2+800 $\mathrm{m}$ (19.2 $\mathrm{mm}$ in unsupported and $16 \mathrm{~mm}$ in supported condition) and minimum displacement along chainage $0+876-1+000 \mathrm{~m}$ (1.4 $\mathrm{mm}$ in unsupported and $1.3 \mathrm{~mm}$ in supported condition). Hence, the estimated support by empirical methods has been optimized by using numerical analyses for the stability of rock mass along the tunnel.
\end{abstract}

\section{Keywords}

Tunnel, Rock Squeezing, Rock Burst, Rock Mass Characterization, Numerical Analyses 


\section{Introduction}

The development of a country is directly related to the energy production. Unfortunately, Pakistan is facing worst energy crisis at present time. The basic and cheapest source of power production is hydropower in Pakistan due to the presence of natural topography which creates natural hydraulic heads along streams especially in hilly areas. Rock bursting is a common and serious form of disasters that can happen in deep underground excavations. The underground excavation passes through variable rock cover, this variation can induce instabilities like spalling, raveling, squeezing and rock bursting. In weak strata squeezing of rocks can take place and rock bursting can occur in un-jointed massive strata where rock mass strength is less than induced stresses [1]. The estimation of rock squeezing, bursting and deformation modulus of rock mass before excavation is one of the most important parameters of the rock mass to mitigate the chances of rock bursting in the excavation i.e. tunnel by applying safest and economical support with both empirical and numerical approaches. In China, during the construction phase of Jinping-II Hydropower project, hundreds of rock bursts occurred which caused damage to the structure, causalities and serious economic loss [2]. In the Sunjiawan Coal mine in 2005, a rock burst caused 215 dead and 30 people injured. Many deep tunnels in China, Canada, Switzerland, and Peru have experienced rock bursting of various degrees. Therefore, the assessment of rock bursting at pre-feasibility and feasibility stage is highly necessary to minimize the casualties. Zhang et al. [3] described the intense rockbursts in tunnels of Jingping II hydropower station by surveying the geological conditions and failure of the affected sections. Gong et al. [4] discussed the problems encountered due to in situ stresses etc. during TBM tunneling and suggested measures to overcome the problems. Kaya et al. [5] investigated the main cause of the failure occurrence mechanism at tunnel portal to determine the remedial measures. Several researchers [6] [7] [8] studied the geological conditions of rock mass to determine the estimated required support alignment by using Rock mass rating (RMR) and Tunneling quality index (Q-system) during excavation along tunnel. Panthee et al. [9] stated that the information of deformation modulus is also required in numerical modeling for underground excavations. The field tests i.e. Dilatometry, plate load tests, flat jack etc. to determine the deformation modulus are time-consuming, expansive and have more chances of error in readings during measurement [10] [11] [12] [13] [14]. Therefore, several researchers suggested empirical equations to determine the rock mass deformation modulus indirectly from correlations with empirical classification systems [15]-[21]. Many researchers [9] [10] [13] [14] [15] [19] [20] [22]-[27] have been studied deformation modulus with empirical equations on the basis of empirical classification systems as a input parameters such as Rock Quality Designation (RQD), Rock Mass Rating (RMR), Q-system (Q), Rock Mass Index (RMi) and Geological Strength Index (GSI).

The study area is located on Ushuriver, Swat valley, Khyber Pakhtunkhwa 
(KP), Pakistan (Figure 1). Ushuriver is a tributary of Swat River in the north of Kalam which is major tourist center in the Swat valley. The geological mapping, discontinuity surveys, and laboratory testing were conducted to classify rock mass by empirical classification systems i.e. RMR, Q, and RMi. Whereas, rock bursting, squeezing and deformation modulus of rock mass along tunnel route were assessed by empirical equations proposed by various researchers. In addition to that numerical analysis along proposed tunnel route was carried out using $\mathrm{RS}^{3}$ [28] in which displacement of rocks in all zones was calculated and advised support categories were assessed by implementing in the model to stable the rock mass during excavation. The detailed methodology of research work is given in Figure 2.

\section{Geological Settings along Tunnel Alignment}

Total three rock units ranging in age from Mesozoic to Cenozoic Era are exposed along tunnel alignment (Figure 3(a)). The northern portion of study area consists of granodiorite while the southern part consists of meta-sediments mainly phyllites, schists, and slates. The meta-sediments have a uniform strike NE-SW and NW dip direction which is fairly steep and less steep towards the north. Granodiorite is grey, greenish grey, medium to coarse grained mainly composed of plagioclase, hornblende, and biotite. Where asschists/phyllites are grey, green in color, thin-bedded, occasionally silty with light grey thin-bedded limestone and quartzite is light to dark grey on the fresh surface and brownish grey on the weathered surface, thin to thick bedded and cherty at places. The soil units are divided as glacio-fluvial deposits, scree, slope wash that is thick at weir site as well as near powerhouse area. The terraces in study area comprised of Glacio-fluvial deposits, which consists of sub-angular to rounded gravels embedded in silt and clay. Scree and slope wash comprised of weathered, disintegrated material due to gravity lying on the toe of the slope. The geological cross section along tunnel presents the rock units intersecting along tunnel alignment shown in Figure 3(b).

\section{Geomechanical Classification along Tunnel Route}

In the empirical analysis, three methods i.e. rock mass rating (RMR), tunneling quality index $(\mathrm{Q})$ and rock mass index (RMi) were used to classify rock mass. The total area of the tunnel was divided into eight segments and all required parameters (orientation, spacing, opening, roughness, the degree of weathering, filling, and groundwater conditions) were collected from each segment to quantify the rock mass by using empirical classification systems. The discontinuity data was plotted on DIPS [29] to assess the pattern of discontinuities and joint sets. In Figure 4, three plots are showing joints distribution in lithological units of granodiorite, quartzite, and phyllite.

Rock quality designation (RQD) was calculated with the help of joint volumetric count $\left(\mathrm{J}_{\mathrm{v}}\right)$ by a relationship given by Palmstrom [30] (Equation (1)). $\mathrm{J}_{\mathrm{v}}$ 


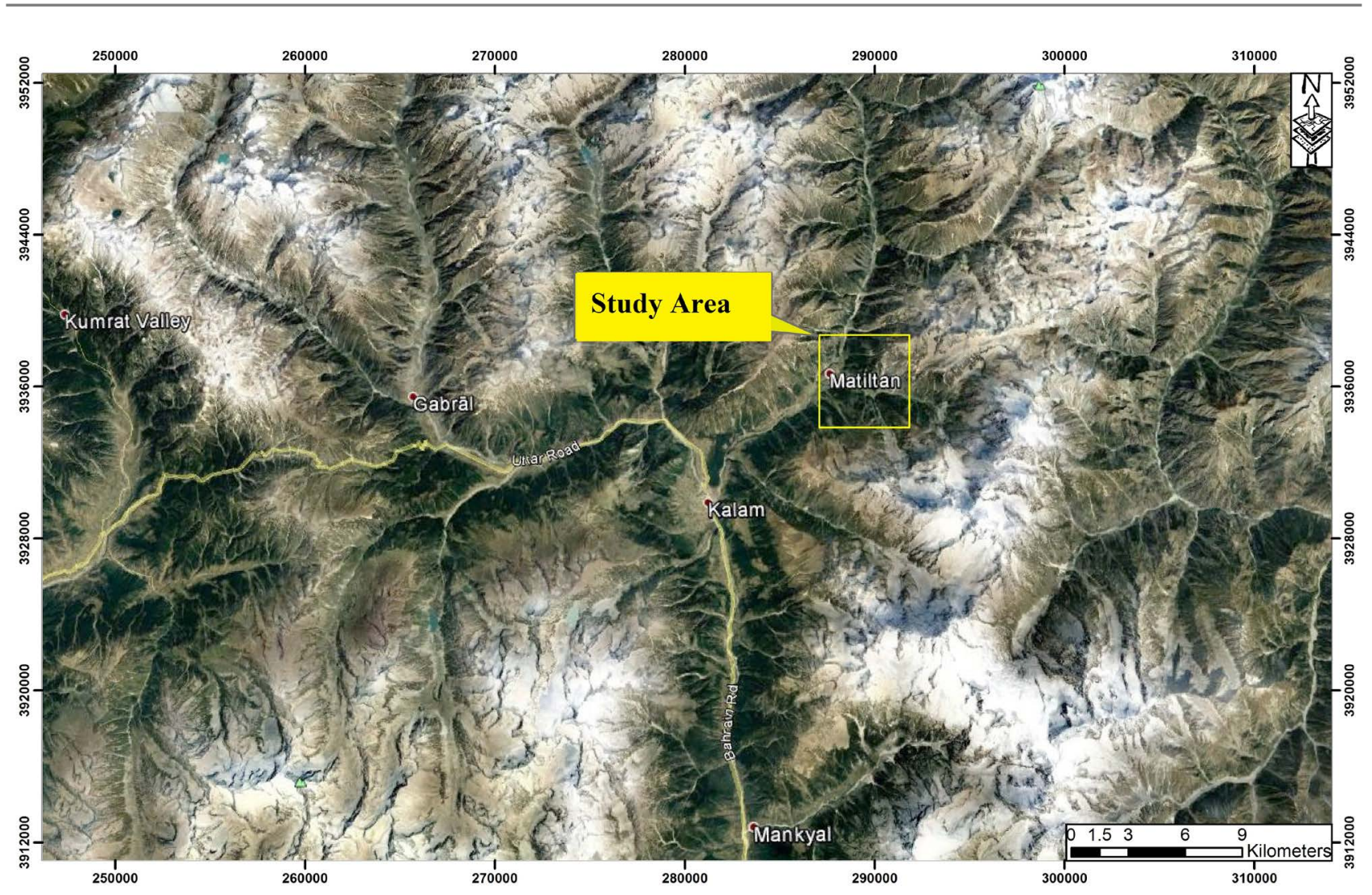

Figure 1. Location of the study area.

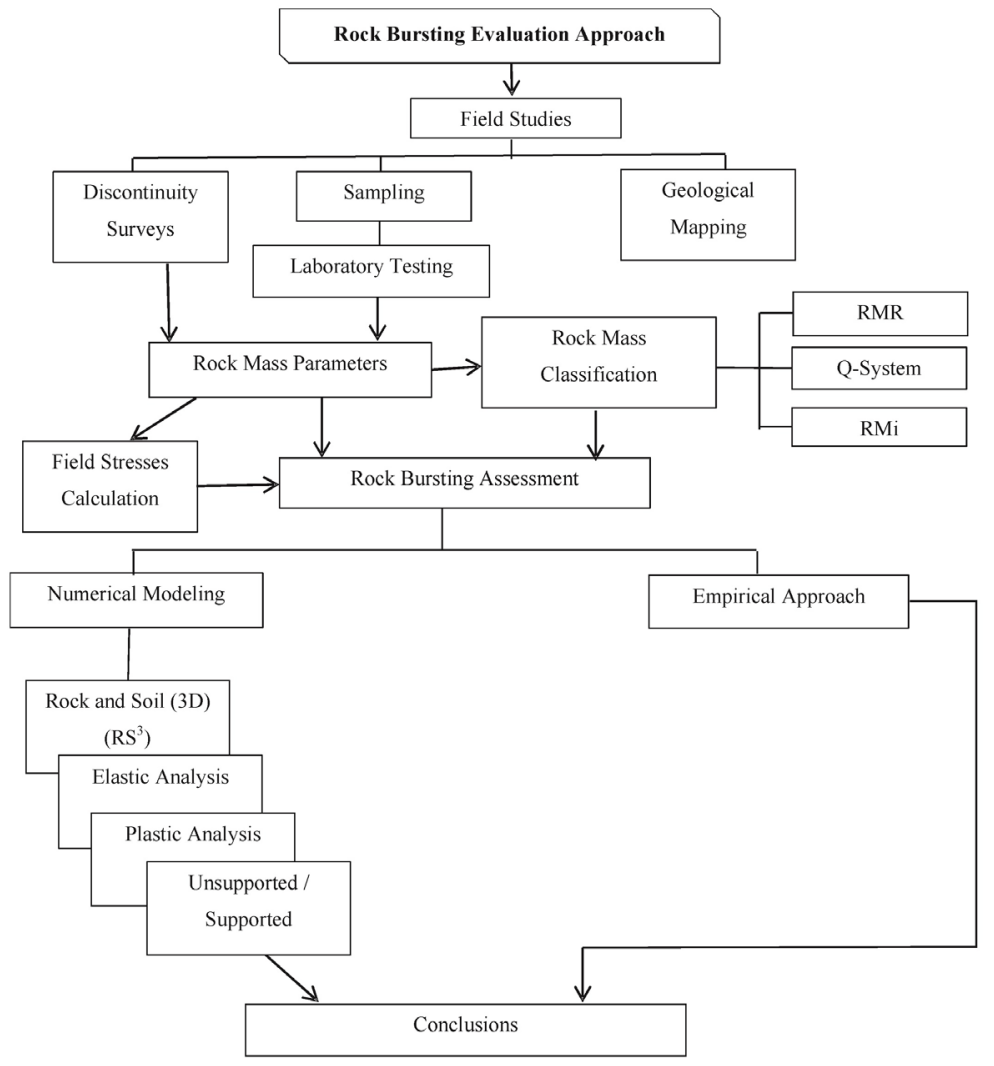

Figure 2. Flow chart of research methodology. 


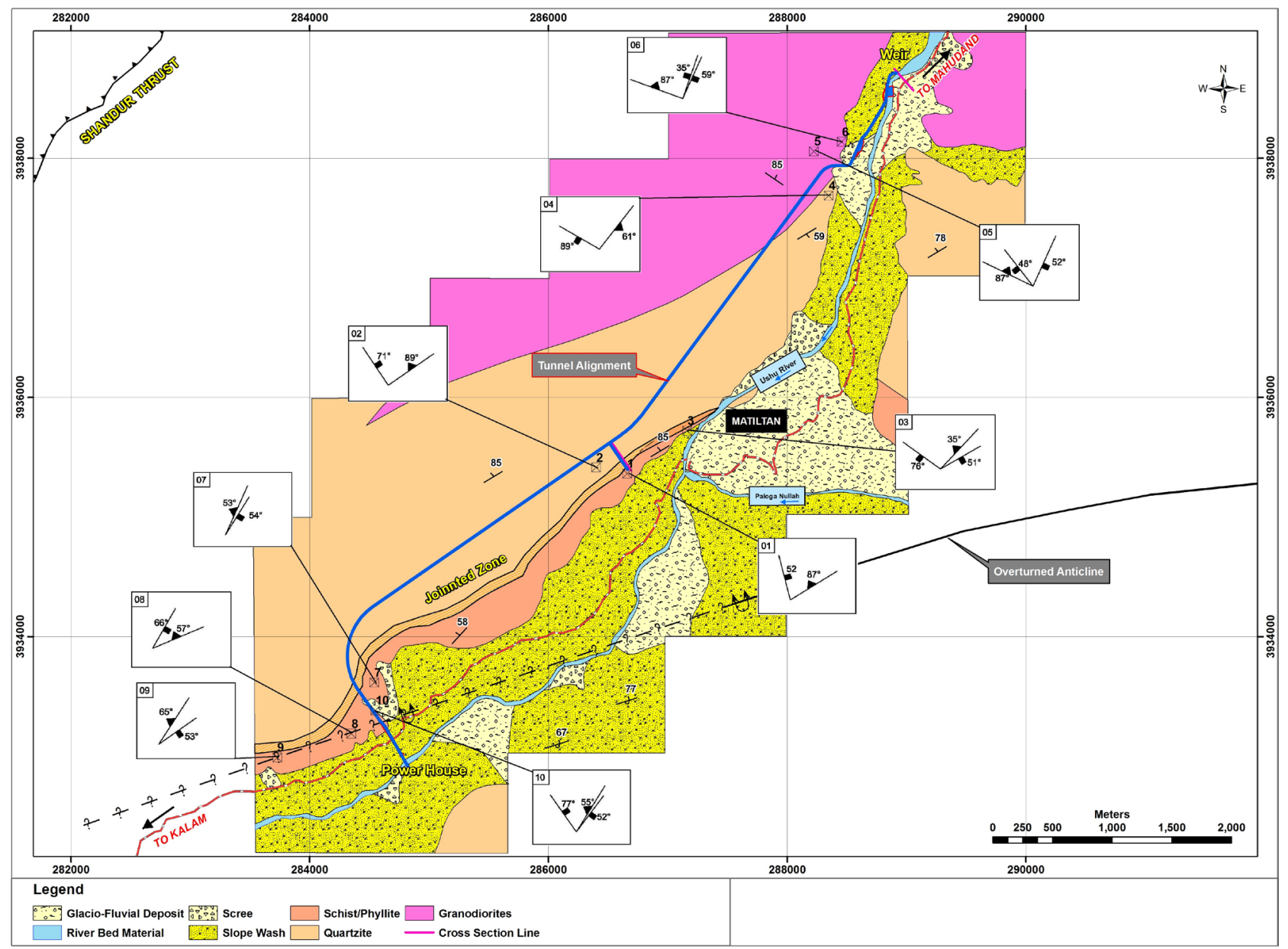

(a)

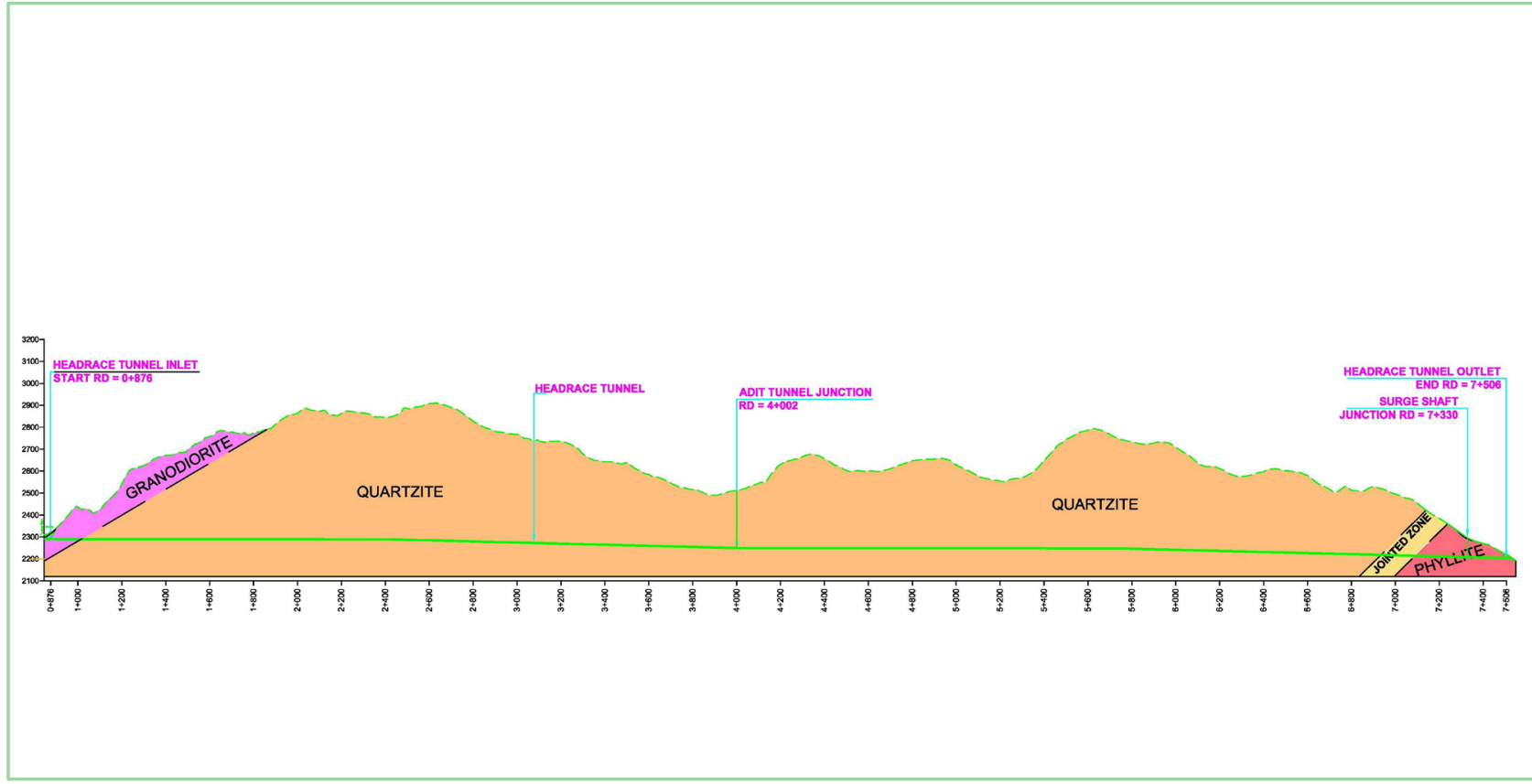

(b)

Figure 3. (a) Engineering geological map of study area; (b) Geological cross section along tunnel route. 


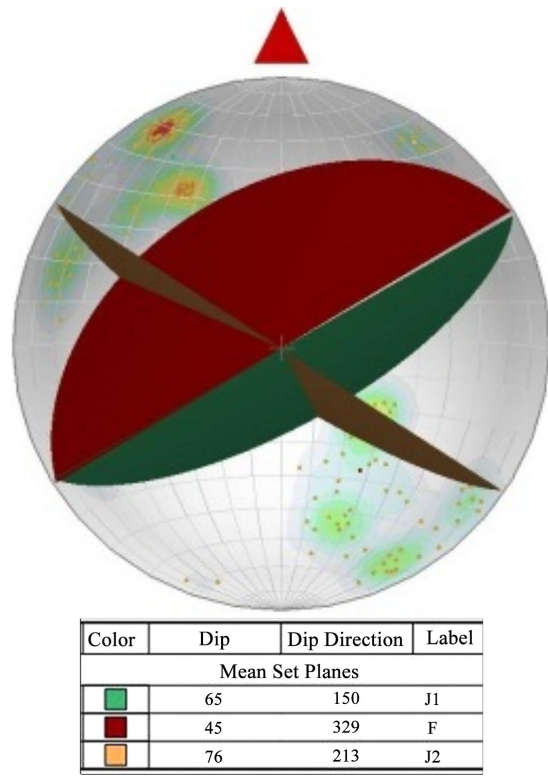

(a)



(b)

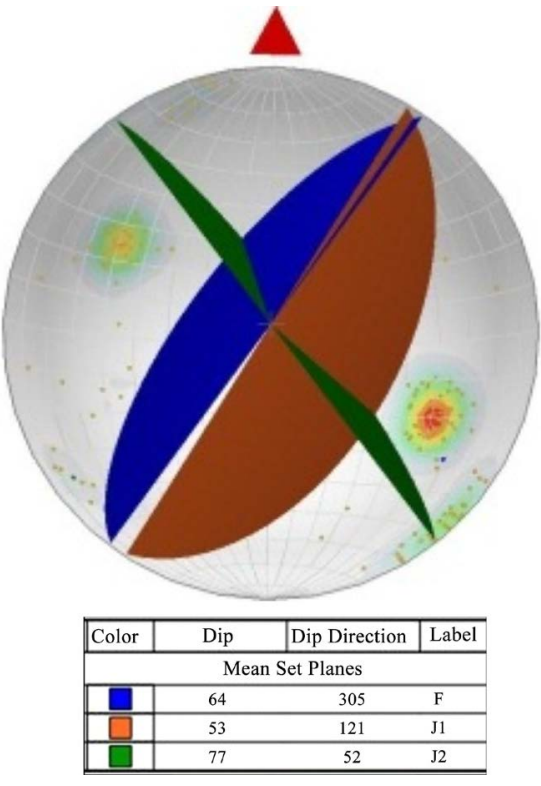

(c)

Figure 4. Plots of discontinuities in 3D view for different rock strata (a) Quartzite; (b) Granodiorite; (c) Phyllite.

represents the total number of joints per cubic meters which are calculated with the help of spacing of joints by following relationship given by Palmstorm [31] [32] [33] [34].

$$
\begin{aligned}
R Q D & =110-2.5 J_{v} \\
J_{v} & =\sum_{i=1}^{J}\left(\frac{1}{S_{i}}\right)
\end{aligned}
$$

where $S_{i}$ is the average joint spacing in meters for the $i^{\text {th }}$ joint and $J$ is the total number of joint sets.

Bieniawski [35] [36] proposed Rock mass rating (RMR) to classify the rock mass. The RMR was calculated by summing all the ratings of six factors: the uniaxial compressive strength of the intact rock, RQD, joints spacing, joints condition, joints orientation. Whereas, tunneling quality index $(\mathrm{Q})$ proposed by Barton et al. [37] that include six parameters as mentioned in Equation (3): RQD, the function of joint sets $(J n)$, discontinuity roughness $(J r)$, joint alteration (Ja), water pressure $(J W)$ and stress reduction factor $(S R F)$. Rock mass index (RMi) is avolumetric parameter and expresses the relative strength of rock mass [38]. In Equation (4) $q_{c}$ represents the uniaxial compressive strength of intact rock and joint parameter $\left(J_{P}\right)$ presents the block volume $(V b)$ plus the joint condition $(j C)$. The $j C$ was measured by joint size $(j L)$, joint alteration $(\mathrm{jA})$ and joint roughness $j R$. The detailed ratings for each parameter of RMR, Q and RMi are summarized in Tables 1-3.

$$
\begin{gathered}
Q=\left[R Q D / J_{n}\left\lceil J_{r} / J_{a}\left\lceil J_{w} / S R F\right]\right.\right. \\
R M i=q c \cdot J P
\end{gathered}
$$

The calculated RMR ratings along chainage $0+876-7+000$ presented fair rock 
quality and along $7+000-7+506$ shows poor rock quality. The $\mathrm{Q}$ values gave fair quality rock at tunnel inlet $(0+876-1+000)$, poor rock along chainage $1+000-$ $7+000$ and very poor quality rock along the chainage $7+000-7+506$ of tunnel alignment. While rock class in RMi is slightly differed from rock class by RMR and Q. RMi shows the strong quality of rock along chainage $0+876-7+000$ and medium quality rock for chainage $7+000$ to $7+506$ of tunnel alignment. By comparing calculated rock mass quality by all three systems it is concluded that rock mass from $0+876$ to $7+000$ lies in the fair quality of rock and along chainage $7+000$ to $7+506$ poor or very poor rock quality depicted. Along chainage $7+000$ to $7+200$, systems presented poor rock quality due to wide jointed and transition zone. Similarly, phyllite intersects the tunnel at chainage $7+200$ to $7+506$ that gave poor rock quality.

Table 1. Quantitative ratings of rock mass parameters according to RMR.

\begin{tabular}{|c|c|c|c|c|c|c|c|c|}
\hline Chainage & $0+876-1+000$ & $1+000-2+800$ & $2+800-4+000$ & $4+000-5+600$ & $5+600-6+600$ & $6+600-7+000$ & $7+000-7+200$ & $7+200-7+506$ \\
\hline UCS & 7 & 7 & 7 & 7 & 7 & 7 & 7 & 2 \\
\hline RQD & 20 & 20 & 20 & 20 & 20 & 20 & 17 & 3 \\
\hline Spacing & 10 & 10 & 10 & 15 & 10 & 10 & 10 & 10 \\
\hline Persistence & 1 & 0 & 0 & 1 & 2 & 1 & 0 & 2 \\
\hline Aperture & 1 & 1 & 0 & 1 & 1 & 1 & 0 & 3 \\
\hline Roughness & 5 & 3 & 1 & 1 & 1 & 3 & 1 & 3 \\
\hline Infilling & 2 & 2 & 6 & 2 & 2 & 2 & 4 & 1 \\
\hline Weathering & 5 & 5 & 5 & 5 & 3 & 3 & 3 & 3 \\
\hline Ground water flow & 7 & 10 & 7 & 10 & 10 & 10 & 4 & 10 \\
\hline Orientation Factor & 0 & -12 & -12 & -12 & -12 & 0 & -12 & 0 \\
\hline Total RMR Rating & 58 & 46 & 44 & 50 & 44 & 57 & 34 & 37 \\
\hline Rock class/Quality & iii/Fair & iii/Fair & iii/Fair & iii/Fair & iii/Fair & iii/Fair & iv/Poor & iv/Poor \\
\hline
\end{tabular}

Table 2. Quantitative ratings for rock mass parameters according to Q system.

\begin{tabular}{|c|c|c|c|c|c|c|c|c|}
\hline Chainage & $0+876-1+000$ & $1+000-2+800$ & $2+800-4+000$ & $4+000-5+600$ & $5+600-6+600$ & $6+600-7+000$ & $7+000-7+200$ & $7+200-7+506$ \\
\hline RQD & 93.61 & 99.8 & 96.1 & 99.81 & 99.65 & 98.21 & 80 & 20.97 \\
\hline Jn & 9 & 4 & 9 & 4 & 4 & 4 & 12 & 9 \\
\hline $\mathrm{RQD} / / n$ & 10.40 & 24.95 & 10.68 & 24.95 & 24.91 & 24.55 & 6.67 & 2.33 \\
\hline$J r$ & 3 & 3 & 3 & 2 & 1.5 & 1.5 & 2 & 3 \\
\hline Ja & 1 & 2 & 2 & 2 & 2 & 2 & 4 & 2 \\
\hline Jr/Ja & 3 & 1.5 & 1.5 & 1 & 0.75 & 0.75 & 0.5 & 1.5 \\
\hline$J w$ & 0.5 & 0.5 & 0.5 & 0.5 & 0.5 & 0.5 & 0.5 & 0.5 \\
\hline SRF & 2.5 & 10 & 5 & 5 & 5 & 2.5 & 5 & 5 \\
\hline$J W / S R F$ & 0.2 & 0.05 & 0.1 & 0.1 & 0.1 & 0.2 & 0.1 & 0.1 \\
\hline Q-Ratings & 6.24 & 1.87 & 1.60 & 2.50 & 1.87 & 3.68 & 0.33 & 0.35 \\
\hline Rock class & Fair & Poor & Poor & Poor & Poor & Poor & Very Poor & Very Poor \\
\hline
\end{tabular}


Table 3. Quantitative ratings for the parameters of rock mass to determine the RMi values along tunnel alignment.

\begin{tabular}{|c|c|c|c|c|c|c|c|c|}
\hline Chainage & $0+876-1+000$ & $1+000-2+800$ & $2+800-4+000$ & $4+000-5+600$ & $5+600-6+600$ & $6+600-7+000$ & $7+000-7+200$ & $7+200-7+506$ \\
\hline UCS & 75 & 65 & 65 & 65 & 65 & 65 & 65 & 15 \\
\hline Block volume $(V b)$ & 0.47 & 0.8 & 0.79 & 1.20 & 1.42 & 0.56 & 0.064 & 0.02 \\
\hline Joint roughness $(J r)$ & 2 & 2 & 1 & 1 & 1 & 2 & 0.5 & 2 \\
\hline Joint length $(j L)$ & 0.75 & 0.75 & 0.75 & 0.75 & 1 & 0.75 & 0.75 & 0.75 \\
\hline Joint alteration $(j A)$ & 2 & 3 & 2 & 2 & 3 & 2 & 4 & 3 \\
\hline Condition factor $(j C)$ & 0.75 & 0.50 & 0.38 & 0.38 & 0.33 & 0.75 & 0.09 & 0.50 \\
\hline $\mathrm{D}$ & 0.39 & 0.43 & 0.45 & 0.45 & 0.46 & 0.39 & 0.59 & 0.43 \\
\hline $\begin{array}{c}\text { Jointing parameter } \\
\qquad(J p)\end{array}$ & 0.13 & 0.13 & 0.11 & 0.13 & 0.14 & 0.14 & 0.01 & 0.03 \\
\hline Rmi ratings & 9.66 & 8.36 & 7.16 & 8.64 & 8.82 & 8.97 & 0.78 & 0.40 \\
\hline Rock class & Strong & Strong & Strong & Strong & Strong & Strong & Medium & Medium \\
\hline
\end{tabular}

\section{Prediction of Ground Condition}

The ground conditions were also assessed by using tunneling quality index values. Singh et al. [39] suggested an empirical approach based on case histories and collected data based on rock mass quality (Q), overburden $(\mathrm{H})$ and developed relations to determine the squeezing (Equation (5)) and non-squeezing (Equation (6)) of the ground. Similarly, Goel et al. [40] proposed an empirical relation based on rock mass number $(\mathrm{N})$ that defined as stress free tunneling quality index $(\mathrm{Q})$, which is used to avoid the problems and uncertainties in obtaining the correct values. The parameters to calculate the $\mathrm{N}$ (Equation (7)) are a tunnel tunneling quality index $(\mathrm{Q})$ and tunnel span or diameter $(\mathrm{B})$ to determine the squeezing (Equation (8)) and non-squeezing (Equation (9)) conditions of the ground.

$$
\begin{gathered}
H \gg 350 Q^{1 / 3} \text { meters } \\
H \ll 350 \mathrm{Q}^{1 / 3} \text { meters } \\
N=(Q)_{S R F=1} \\
H \gg 275 N^{0.33} \cdot B^{-1}(m) \\
H \ll 275 N^{0.33} \cdot B^{-1}(m)
\end{gathered}
$$

The values calculated with relation proposed by Singh et al. [39] in which SRF value of 2.5 were used that is presented in Table 4 and plotted in Figure 5(a) that shows whole tunnel lies in non-squeezing zone. Similarly, according to the approach of Goel et al. [40] the calculated values are listed in Table 4 that lies on the line $A B$ (Figure $5(b)$ ). So, the rock units were predicted in minor to non-squeezing conditions.

Sengupta [41] proposed relations of Equations (10) and (11) to calculate the field stresses for overburden less than $400 \mathrm{~m}$ and Stephansson [42] suggested the 
relation (Equations (12) and (13)) for overburden less than $1000 \mathrm{~m}$. In anisotropic stress conditions, the tangential stresses vary around the periphery of the opening. According to Kirsch's solution (Equations (15) and (16)), tangential stress will reach its maximum value where maximum principal stress $\left(\sigma_{1}\right)$ is $\tan$ gent to the excavation contour and minimum tangential stress value where its minimum principal stress is tangent to excavation contour. Hoek and Brown [42] proposed a method to estimate the tangential stresses for roof $\left(\sigma_{\theta r}\right)$ and walls ( $\sigma_{\theta w}$ in massive rocks according to excavation shapes (Equations (17) and (18)). The calculated values of field stresses are given in Table 5.

$$
\begin{gathered}
\sigma_{1}=\sigma_{H}=1.5+1.2 \sigma_{v}(\mathrm{MPa}) \\
\sigma_{3}=\sigma_{h}=1.0+0.5 \sigma_{v}(\mathrm{MPa}) \\
\sigma_{1}=\sigma_{H}=2.8+1.48 \sigma_{v}(\mathrm{MPa}) \\
\sigma_{3}=\sigma_{h}=2.2+0.89 \sigma_{v}(\mathrm{MPa}) \\
\sigma_{v}=\gamma Z \\
\sigma_{\theta \max }=3 \sigma_{1}-\sigma_{3} \\
\sigma_{\theta \min }=3 \sigma_{3}-\sigma_{1} \\
\sigma_{\theta r}=(A \times k-1) \sigma_{z} \\
\sigma_{\theta w}=(B-k) \sigma_{z}
\end{gathered}
$$

where $\sigma_{\theta}$ is for tangential stress ( $\sigma_{\theta r}$ for roof and $\sigma_{\theta w}$ for wall), $k$ is the horizontal/vertical stress ratio, $\sigma_{\mathrm{z}}$ is the vertical stress and $\mathrm{A}, \mathrm{B}$ are the excavation geometry factors.

Moreover, several other approaches developed by researchers i.e. [42] [43] [44] [45] were used to assess rock bursting potential (Table 6) by using field stresses (Major, minor, intermediate, tangential stress). Hoek and brown [42] have made detail studies for the stability analysis in different tunnels in South Africa. The ratio of uniaxial compressive strength $\left(\sigma_{\mathrm{c}}\right)$ and tangential stress $\left(\sigma_{\Theta}\right)$ was compared in this study to assess the rock bursting condition. Grimstad and Barton [44] made a relation by using stress measurements, the strength of the rocks and arrived at relationships which also support the findings of Hoek and Brown [42]. They also described the bursting potential by using the ratio of compressive strength and tangential stress $\left(\sigma_{\mathrm{c}} / \sigma_{\Theta}\right)$ as mentioned in Table 6. The detail results of rock bursting assessment by both researchers are given in Table 7. Palmstorm [45] used the rock mass index (RMi) that expresses the relative compressive strength of rock mass and tangential stress $\left(\sigma_{\Theta}\right)$ to assess the competency factor $(\mathrm{Cg})$ as mentioned in Equation (19).

$$
\mathrm{Cg}=R M i / \sigma_{\theta}
$$

Russenes (1974) devised a relationship of point load strength $\left(I_{s(50)}\right)$ of rocks and tangential stresses to assess the rock bursting during excavation. Point load strength was calculated according to the instructions of ASTM D 5731 - 95 [46] 
standard method. The tangential stress was calculated from the maximum and minimum principal stress. The acquired values were plotted in the graph produced by Russenes (Figure 6) and it was noted from Table 8 that chainage $1+000-3+600,5+200-5+600$ lies in moderate rock burst and $0+876-1+000$, $3+600-5+200,5+600-7+506$ depicts no rock burst activity. The equations proposed by Palmstrom and Singh [10] (Equation (20)) and Read et al. [47] (Equation (21)) were used to estimate deformation modulus $\left(E_{\mathrm{m}}\right)$ in which RMR and $\mathrm{Q}$ values considered as input parameters. The calculated values of $E_{\mathrm{m}}$ along tunnel route are presented in Table 9.

$$
\begin{array}{r}
E_{m}(\mathrm{GPa})=8 Q^{0.4} \\
E_{m}(\mathrm{GPa})=0.1(R M R / 10)^{3}
\end{array}
$$

\begin{tabular}{|c|c|c|c|c|c|c|c|c|c|}
\hline \multirow{2}{*}{ Chainage } & \multirow{2}{*}{ Overburden (m) } & \multicolumn{3}{|c|}{$\begin{array}{l}\text { By relation proposed by } \\
\text { Singh et al. }\end{array}$} & \multicolumn{5}{|c|}{$\begin{array}{l}\text { By relation proposed by } \\
\text { Goel et al. }\end{array}$} \\
\hline & & Q-value & $\mathrm{H}$ & Condition & $\mathrm{N}$ & B & $\mathrm{H}$ & $\mathrm{HB}^{0.1}$ & Condition \\
\hline $0+876-1+000$ & 165 & 6.24 & 640.46 & Non-Squeezing & 15.6 & & 579.65 & 680.87 & $\begin{array}{l}\text { Non-Minor } \\
\text { Squeezing }\end{array}$ \\
\hline $1+000-1+600$ & 400 & & & & & & & & \\
\hline $1+600-2+000$ & 530 & & & & & & & & Non-Minor \\
\hline $2+000-2+400$ & 575 & 7.49 & 680.07 & Non-Squeezing & 18.71 & & 615.49 & 722.96 & Squeezing \\
\hline $2+400-2+800$ & 600 & & & & & & & & \\
\hline $2+800-3+200$ & 440 & & & & & & & & \\
\hline $3+200-3+600$ & 430 & 3.20 & 513.95 & Non-Squeezing & 8.01 & & 465.19 & 546.43 & $\begin{array}{l}\text { Non-Minor } \\
\text { Squeezing }\end{array}$ \\
\hline $3+600-4+000$ & 250 & & & & & & & & \\
\hline $4+000-4+400$ & 285 & & & & & & & & \\
\hline & & & & & & 5 & & & \\
\hline $\begin{array}{l}4+400-4+800 \\
4+800-5+200\end{array}$ & 310 & 4.99 & 594.92 & Non-Squeezing & 12.48 & & 538.5 & 632.53 & $\begin{array}{c}\text { Non-Minor } \\
\text { Squeezing }\end{array}$ \\
\hline $5+200-5+600$ & 455 & & & & & & & & \\
\hline $\begin{array}{l}5+600-6+000 \\
6+000-6+600\end{array}$ & 380 & 3.74 & 540.75 & Non-Squeezing & 9.34 & & 489.38 & 574.84 & $\begin{array}{c}\text { Non-Minor } \\
\text { Squeezing }\end{array}$ \\
\hline $6+600-7+000$ & 265 & 3.68 & 538.16 & Non-Squeezing & 9.21 & & 487.13 & 572.19 & $\begin{array}{c}\text { Non-Minor } \\
\text { Squeezing }\end{array}$ \\
\hline $7+000-7+200$ & 145 & 0.67 & 306.17 & Non-Squeezing & 1.67 & & 277.29 & 325.71 & $\begin{array}{l}\text { Non-Minor } \\
\text { Squeezing }\end{array}$ \\
\hline $7+200-7+506$ & 40 & 0.7 & 310.99 & Non-Squeezing & 1.75 & & 281.6 & 330.78 & $\begin{array}{c}\text { Non-Minor } \\
\text { Squeezing }\end{array}$ \\
\hline
\end{tabular}

Table 4. Prediction of ground conditions by using empirical relations. 
Table 5. Estimated field stresses of rock mass by using rock mass parameters and relations developed by various researchers.

\begin{tabular}{|c|c|c|c|c|c|c|c|c|}
\hline \multirow{3}{*}{ Chainage } & \multirow{3}{*}{$\begin{array}{c}\text { Overburden }(\mathrm{H}) \\
\mathrm{m}\end{array}$} & \multirow{2}{*}{ Unit weight $(\gamma)$} & \multicolumn{6}{|c|}{ Stresses } \\
\hline & & & $\sigma_{1}$ & $\sigma_{2}$ & $\sigma_{3}$ & $\sigma_{\theta}$ & $\sigma_{\text {er }_{\mathrm{r}}}$ & $\sigma_{\theta_{\mathrm{w}}}$ \\
\hline & & $\mathrm{MN} / \mathrm{m}^{3}$ & \multicolumn{6}{|c|}{$\mathrm{MPa}$} \\
\hline $0+876-1+000$ & 165 & 0.027 & 6.84 & 4.45 & 3.22 & 10.06 & 9.78 & 5.78 \\
\hline $1+000-1+600$ & 400 & 0.027 & 18.75 & 11.79 & 10.78 & 30.55 & 26.96 & 13.00 \\
\hline $1+600-2+000$ & 530 & 0.027 & 23.94 & 14.91 & 14.28 & 38.85 & 33.44 & 17.94 \\
\hline $2+000-2+400$ & 575 & 0.027 & 25.73 & 15.99 & 15.50 & 41.73 & 35.68 & 19.65 \\
\hline $2+400-2+800$ & 600 & 0.027 & 26.73 & 16.59 & 16.17 & 43.32 & 36.92 & 20.60 \\
\hline $2+800-3+200$ & 440 & 0.027 & 20.35 & 12.75 & 11.86 & 33.10 & 28.95 & 14.52 \\
\hline $3+200-3+600$ & 430 & 0.027 & 19.95 & 12.51 & 11.59 & 32.46 & 28.46 & 14.14 \\
\hline $3+600-4+000$ & 250 & 0.027 & 9.59 & 6.74 & 4.37 & 13.95 & 7.24 & 11.13 \\
\hline $4+000-4+400$ & 285 & 0.027 & 10.72 & 7.68 & 4.84 & 15.56 & 7.81 & 12.83 \\
\hline $4+400-4+800$ & 310 & 0.027 & 11.53 & 8.35 & 5.18 & 16.70 & 8.21 & 14.04 \\
\hline $4+800-5+200$ & 295 & 0.027 & 11.04 & 7.95 & 4.98 & 16.02 & 7.97 & 13.31 \\
\hline $5+200-5+600$ & 455 & 0.027 & 20.95 & 13.11 & 12.26 & 34.06 & 29.70 & 15.09 \\
\hline $5+600-6+000$ & 380 & 0.027 & 13.79 & 10.24 & 6.12 & 19.91 & 9.34 & 17.43 \\
\hline $6+000-6+600$ & 330 & 0.027 & 12.17 & 8.89 & 5.45 & 17.62 & 8.54 & 15.01 \\
\hline $6+600-7+000$ & 265 & 0.027 & 10.07 & 7.14 & 4.57 & 14.64 & 7.49 & 11.86 \\
\hline $7+000-7+200$ & 145 & 0.027 & 6.12 & 3.85 & 2.93 & 9.05 & 5.51 & 5.93 \\
\hline $7+200-7+506$ & 40 & 0.027 & 2.77 & 1.06 & 1.53 & 4.31 & 3.84 & 0.91 \\
\hline
\end{tabular}

Table 6. Description of rock bursting potential relations proposed by Hoek and Brown [42], Grimstad and Barton [44], Palmstrom [45].

\begin{tabular}{|c|c|c|c|c|c|}
\hline \multicolumn{2}{|c|}{ Hoek \& Brown [42] } & \multicolumn{2}{|c|}{ Grimstad \& Barton [44] } & \multicolumn{2}{|c|}{ Palmstorm [45] } \\
\hline Ratio $\left(\sigma_{\mathrm{c}} / \sigma_{\circledast}\right)$ & Description & Ratio $\left(\sigma_{\mathrm{c}} / \sigma_{\circledast}\right)$ & Description & $\begin{array}{l}\text { Competency } \\
\text { Factor }(\mathrm{Cg})\end{array}$ & Failure Modes \\
\hline$\sigma_{\mathrm{c}} / \sigma_{\Theta}>7$ & Stable & $\sigma_{\mathrm{c}} / \sigma_{\Theta}>100$ & $\begin{array}{c}\text { Low stress, near surface, open } \\
\text { joints }\end{array}$ & $<2.5$ & $\begin{array}{l}\text { No rock stress induced } \\
\text { instability }\end{array}$ \\
\hline$\sigma_{\mathrm{c}} / \sigma_{\Theta}=3.5$ & $\begin{array}{l}\text { Minor sidewall } \\
\text { spalling }\end{array}$ & $\sigma_{\mathrm{c}} / \sigma_{\Theta}=3-100$ & $\begin{array}{l}\text { Medium stress, favorable stress } \\
\text { conditions }\end{array}$ & $2.5-1$ & $\begin{array}{l}\text { High stress, slightly } \\
\text { loosening }\end{array}$ \\
\hline$\sigma_{\mathrm{c}} / \sigma_{\Theta}=2$ & Severe spalling & $\sigma_{\mathrm{c}} / \sigma_{\Theta}=2-3$ & $\begin{array}{l}\text { High stress, usually favorable to } \\
\text { stability, maybe } \\
\text { unfavorable to wall stability }\end{array}$ & $1-0.5$ & Light rock burst or spalling \\
\hline$\sigma_{\mathrm{c}} / \sigma_{\Theta}=1.7$ & $\begin{array}{l}\text { Heavy support } \\
\text { required }\end{array}$ & $\sigma_{\mathrm{c}} / \sigma_{\Theta}=1.5-2$ & $\begin{array}{c}\text { Moderate slabbing after one } \\
\text { hour }\end{array}$ & & \\
\hline$\sigma_{\mathrm{c}} / \sigma_{\Theta}<1.4$ & $\begin{array}{l}\text { Severe rock burst } \\
\text { problem }\end{array}$ & $\sigma_{\mathrm{c}} / \sigma_{\Theta}=1-1.5$ & $\begin{array}{l}\text { Slabbing and rock burst after } \\
\text { minutes in massive rocks } \\
\text { Heavy rock burst and } \\
\text { immediate rock deformation }\end{array}$ & $<0.5$ & Heavy rock burst \\
\hline
\end{tabular}


Table 7. Assessment of rock bursting potential on roof and walls of proposed tunnel.

\begin{tabular}{|c|c|c|c|c|c|c|}
\hline \multirow{2}{*}{ Chainage } & \multicolumn{3}{|c|}{ By relation proposed by Hoek and Brown [42] } & \multicolumn{3}{|c|}{ By relation proposed by Grimstad and Barton [44] } \\
\hline & $\sigma_{c} / \sigma_{\circledast}$ & For Roof & For walls & $\sigma_{\mathrm{c}} / \sigma_{\circledast}$ & For Roof & For walls \\
\hline $0+876-1+000$ & 7.67 & Stable & Stable & 12.97 & $\begin{array}{l}\text { Medium stress, Favourable } \\
\text { stress Condition }\end{array}$ & \\
\hline $1+000-1+600$ & 2.41 & Severe spalling & $\begin{array}{l}\text { Minor (Side wall) } \\
\text { spalling }\end{array}$ & 5.00 & $\begin{array}{l}\text { High Stress, Usually } \\
\text { favourable to Stability }\end{array}$ & \\
\hline $1+600-2+000$ & 1.94 & & & 3.62 & & \\
\hline $2+000-2+400$ & 1.82 & $\begin{array}{l}\text { Heavy Support } \\
\text { Required }\end{array}$ & Severe spalling & 3.31 & $\begin{array}{l}\text { Moderate Slabbing after } 1 \\
\text { hour }\end{array}$ & \\
\hline $2+400-2+800$ & 1.76 & & & 3.16 & & \\
\hline $\begin{array}{l}2+800-3+200 \\
3+200-3+600\end{array}$ & 2.24 & Severe spalling & & $\begin{array}{l}4.48 \\
4.60\end{array}$ & $\begin{array}{l}\text { High Stress, Usually } \\
\text { favourable to Stability }\end{array}$ & \\
\hline $3+600-4+000$ & 8.97 & & & 5.84 & & $\begin{array}{l}\text { Medium } \\
\text { stress, }\end{array}$ \\
\hline $\begin{array}{l}4+000-4+400 \\
4+400-4+800\end{array}$ & $\begin{array}{l}8.32 \\
7.91\end{array}$ & Stable & & $\begin{array}{l}5.07 \\
4.63\end{array}$ & $\begin{array}{l}\text { Medium stress, } \\
\text { Favourable stress } \\
\text { Condition }\end{array}$ & $\begin{array}{l}\text { Favourable } \\
\text { stress } \\
\text { Condition }\end{array}$ \\
\hline $4+800-5+200$ & 8.16 & & $\begin{array}{l}\text { Minor (Side wall) } \\
\text { spalling }\end{array}$ & 4.88 & & \\
\hline $5+200-5+600$ & 2.19 & Severe spalling & & 4.31 & $\begin{array}{l}\text { High Stress, Usually } \\
\text { favourable to Stability }\end{array}$ & \\
\hline $5+600-6+000$ & 6.96 & $\begin{array}{l}\text { Minor (Side wall) } \\
\text { spalling }\end{array}$ & & 3.73 & & \\
\hline $6+000-6+600$ & 7.61 & & & 4.33 & Medium stress, & \\
\hline $6+600-7+000$ & 8.68 & Stable & & 5.48 & $\begin{array}{l}\text { Favourable stress } \\
\text { Condition }\end{array}$ & \\
\hline $7+000-7+200$ & 11.80 & & Stable & 10.96 & & \\
\hline $7+200-7+506$ & 3.91 & $\begin{array}{l}\text { Minor (Side wall) } \\
\text { spalling }\end{array}$ & Stable & 16.44 & & \\
\hline
\end{tabular}

Table 8. Assessment for the potential of rock bursting along roof and walls of proposed tunnel by using relations developed by Palmstorm [45] and Russenes [43].

\begin{tabular}{|c|c|c|c|c|c|c|c|}
\hline \multirow{2}{*}{ Chainage } & \multicolumn{4}{|c|}{ Palmstrom [45] } & \multicolumn{3}{|c|}{ Russenes [43] } \\
\hline & $\mathrm{RMi} / \sigma_{\oplus \mathrm{r}}$ & $\mathrm{RMi} / \sigma_{\Theta \mathrm{w}}$ & For Roof & For walls & $I_{s(50)}$ & $\sigma_{\circledast}$ & Description \\
\hline $0+876-1+000$ & 0.99 & 1.67 & Light Rock Burst & $\begin{array}{c}\text { High Stress, } \\
\text { slightly loosening }\end{array}$ & 5.65 & 10.06 & No rock burst \\
\hline $1+000-1+600$ & 0.31 & 0.64 & & Light rock burst & 4.52 & 30.55 & \\
\hline $1+600-2+000$ & 0.25 & 0.47 & & & 4.52 & 38.85 & \\
\hline $2+000-2+400$ & 0.23 & 0.43 & Heavy Rock Burst & t & 4.52 & 41.73 & Moderate rock burst \\
\hline $2+400-2+800$ & 0.23 & 0.41 & & Heavy Kock Burst & 4.52 & 43.32 & \\
\hline $2+800-3+200$ & 0.25 & 0.49 & & & 4.52 & 33.10 & \\
\hline
\end{tabular}


M. S. Akram et al.

\section{Continued}

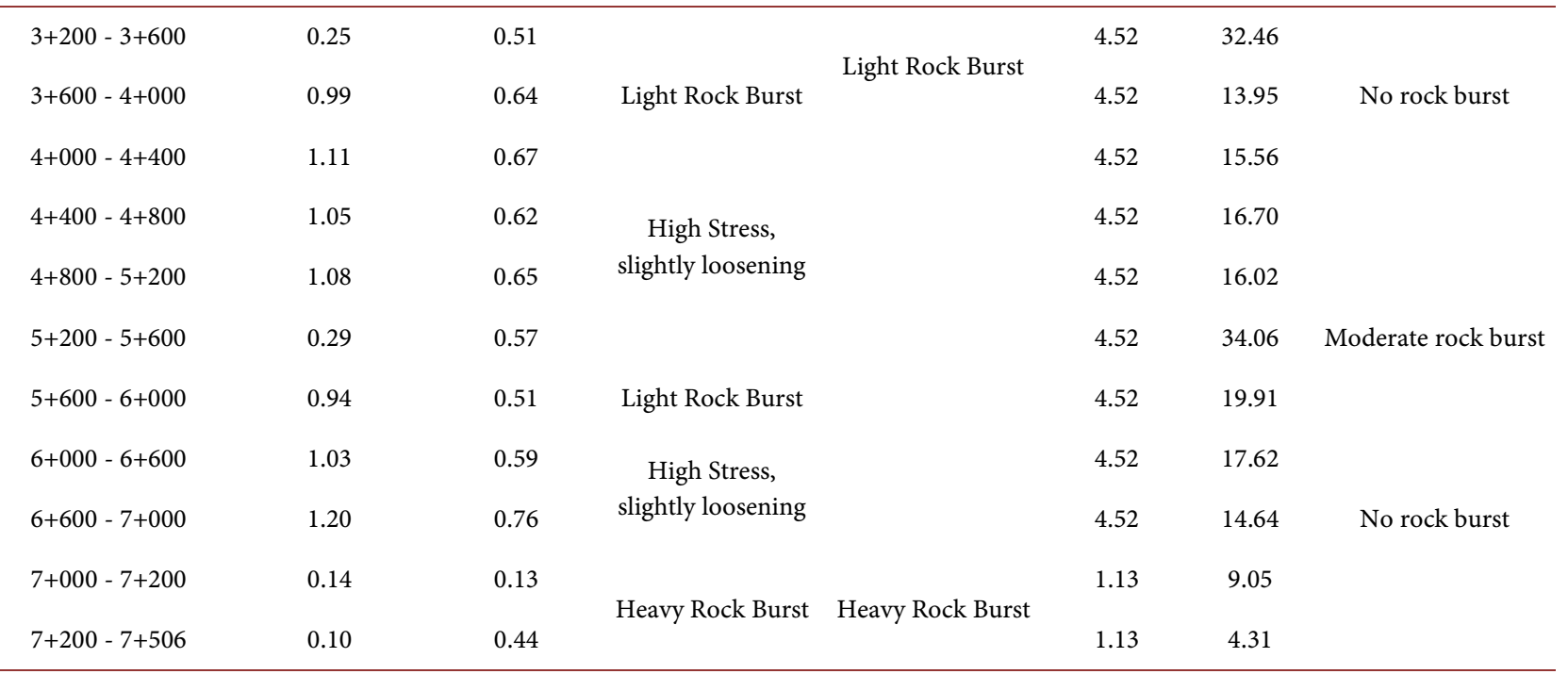

Table 9. Calculated values of deformation modulus $\left(E_{m}\right)$ of rock mass.

\begin{tabular}{cccc}
\hline \multirow{2}{*}{ Chainage } & \multicolumn{2}{c}{ Calculated Values for $E_{m}$} \\
\cline { 3 - 4 } & & Equation (21) & Equation (22) \\
\hline $0+876$ & $1+000$ & 16.64 & 19.51 \\
$1+000$ & $2+800$ & 10.28 & 9.73 \\
$2+800$ & $4+000$ & 9.65 & 8.52 \\
$4+000$ & $5+600$ & 11.54 & 12.50 \\
$5+600$ & $6+600$ & 10.28 & 8.52 \\
$6+600$ & $7+000$ & 13.47 & 18.52 \\
$7+000$ & $7+200$ & 5.13 & 3.93 \\
$7+000$ & $7+506$ & 5.26 & 5.07 \\
\hline
\end{tabular}

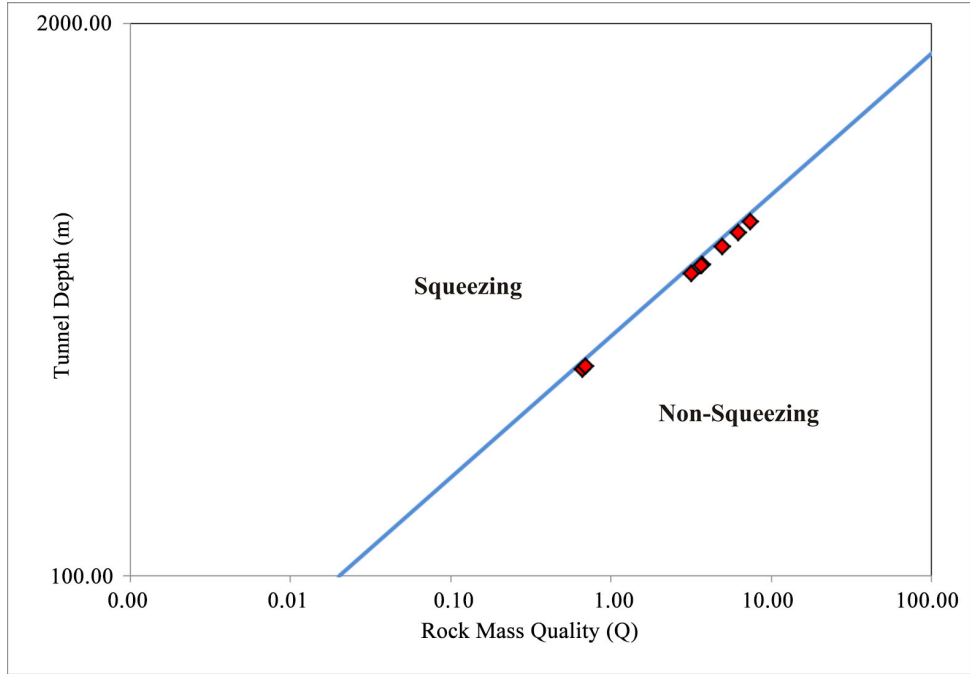

(a) 


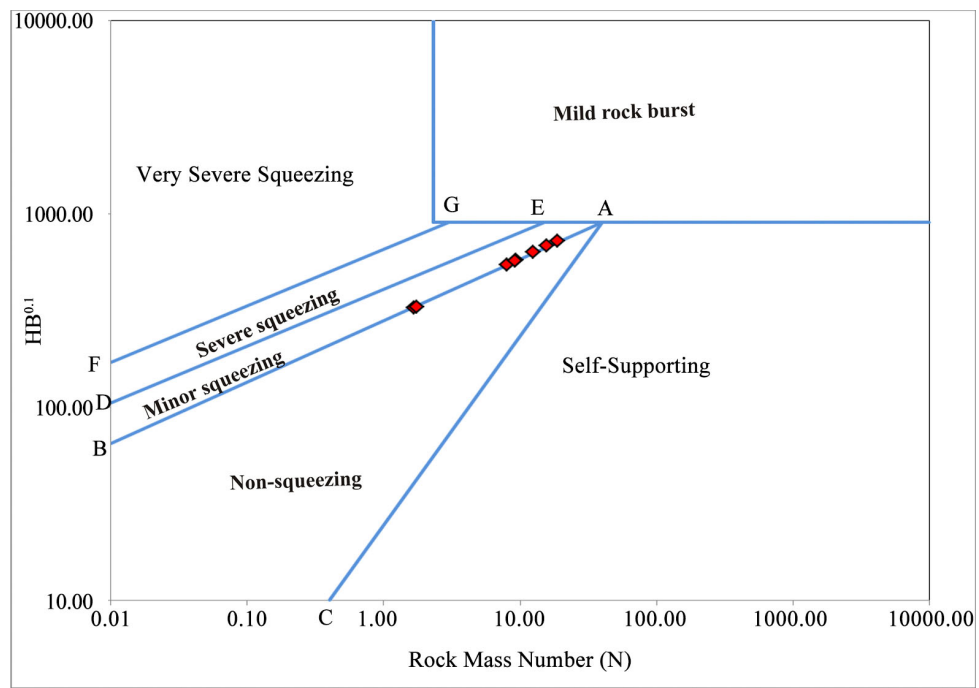

(b)

Figure 5. Graphical representation of ground assessment by empirical relations (modified after Singh et al. [39], Goel et al. [40]).

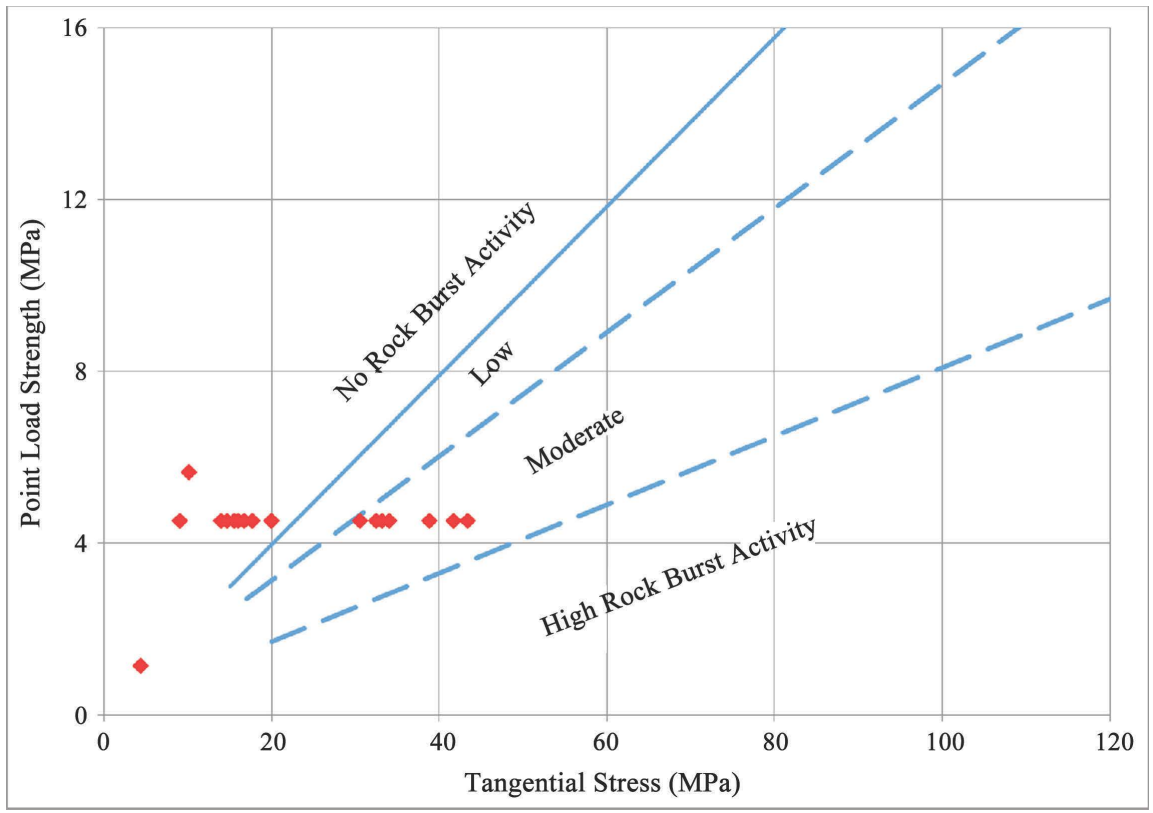

Figure 6. Assessment of rock burst by using point load strength and tangential stress in tunnel (modified after Russenes [42]).

\section{Estimated Rock Support and Numerical Analysis}

The rock quality was classified by the empirical methods RMR, Q, and RMi. Application of these empirical methods includes rock support estimation based on rock quality. In this study, RMR gave fair rock quality along chainage $0+876$ to $7+000$ and poor rock quality for chainage 7+000 - 7+506 and estimated support for these zones of the tunnel is suggested in Table 10. Barton et al. [37] established a chart to estimate support category in which Q-value and equivalent dimension (De) is used. De is calculated by dividing the span of the tunnel with 
equivalent support ratio (ESR). Support category proposed by Barton's Q-system and recommended support is given in Table 10. The calculated support by empirical methods was installed in the $\mathrm{RS}^{3}$ model to control displacement along proposed tunnel alignment.

Panthee et al. [9] stated that use of numerical analyses has become avital partin the planning of engineering projects. Similarly many researchers [9] [48]-[56] have successfully utilized the numerical techniques to sort out the rock engineering problems. This study includes the numerical analyses using computer-aided program $\mathrm{RS}^{3}$ (v. 1.0) to excavate the headrace tunnel in horseshoe-shape with a $5 \mathrm{~m}$ diameter. In 3D model, Hoek and Brown failure criteria was used for isotropic material with aslice thickness of $15 \mathrm{~m}$. There were two types of in-situ stresses used in the model; constant and gravitational stresses. In analyses, constant field stresses were used as deep excavations, where gravitational field stress is negligible across the height of model. The rock mass was allowed to deform both elastically and plastically for analyses. The in-situ vertical and horizontal stresses were assumed by the depth of tunnel. Whereas, the maximum number of iterations 500 for elastic and 1500 for plastic analysis to determine the displacement and rock mass failures. The external boundary was used as abox with anexpansion factor of 3 and a graded mesh with 4 nodded triangles were used for analyses. While a gradation factor of 0.1 and number of excavation nodes 110 were used and discretization of the excavation boundary was determined by the number of excavation nodes. Several models were prepared for every $400 \mathrm{~m}$ Section in different lithology along tunnel alignment. Firstly, support estimated by empirical methods was installed then support was optimized by $\mathrm{RS}^{3}$ model to control the displacement along the tunnel. Optimized support in each section shown in Table 11.

Numerical models are totally dependent on the quality of input parameters. Hence, if there is uncertainty in input parameters then it leads to uncertainty in the analysis. Models are prepared for elastic and plastic ground conditions as shown in Figures 7(a)-(d). Plastic ground condition is a worst scenario where yielding is maximum. Support is installed in both conditions to assess the variation in total displacement. The maximum total displacement along tunnel alignment is at section $2+400-2+800$ having tunnel depth $600 \mathrm{~m}$. Without support, in elastic conditions, total displacement is $14.5 \mathrm{~mm}$ which is reduced to $13.5 \mathrm{~mm}$ after installation of support. In plastic condition, total displacement without support in that zone is $19.2 \mathrm{~mm}$ which is reduced to $16.0 \mathrm{~mm}$ after installation of support. The section of inlet portal of the tunnel (chainage $0+876$ to $1+000$ ) with the depth of $165 \mathrm{~m}$ comprised of massive and jointed granodiorite. In elastic conditions, this section has a total displacement of $1.4 \mathrm{~mm}$ that is reduced to $1.3 \mathrm{~mm}$ after installation of support. Similarly, the section of the tunnel (chainage $1+000$ to $1+600$ ) comprised of quartzite having $400 \mathrm{~m}$ overburden. In elastic conditions, the displacement of $10.4 \mathrm{~mm}$ was noted without the support and after installation of support, the displacement was reduced to $9.7 \mathrm{~mm}$. 
Whereas, the displacement was slightly increased to $12.2 \mathrm{~mm}$ in plastic conditions and reduced to $10.7 \mathrm{~mm}$ after applied support. The tunnel section of chainage $2+400-2+800$ has the maximum depth that results in maximum stresses. In elastic conditions, total displacement without support was $14.5 \mathrm{~mm}$, which is reduced to $13.5 \mathrm{~mm}$ after installation of support and in plastic conditions displacement was reduced from $19.2 \mathrm{~mm}$ to $16.0 \mathrm{~mm}$ after installation of support. The section of the tunnel (chainage 7+000 - 7+200) intersects the jointed zone. In elastic conditions, total displacement was decreased from $10.5 \mathrm{~mm}$ to $8.3 \mathrm{~mm}$ after application of support and the length of the bolts in this zone was increased to $3 \mathrm{~m}$ due to widely spaced joints. In plastic conditions, the total displacement of $10.9 \mathrm{~mm}$ was encountered that was reduced to $8.4 \mathrm{~mm}$ after installation of support. The zone of chainage 7+200 - 7+506 near tunnel outlet intersects the phyllitethat gave displacement is $11.7 \mathrm{~mm}$ that is reduced to $7.8 \mathrm{~mm}$ after support installation in elastic conditions. While in plastic conditions, total displacement was $17.9 \mathrm{~mm}$ that is reduced to $9.6 \mathrm{~mm}$ after installation of support.

Table 10. Estimated support by using classification systems i.e. rock mass rating (RMR) and tunneling quality index (Q).

\begin{tabular}{|c|c|c|c|c|c|}
\hline \multirow{3}{*}{ Chainage } & \multicolumn{2}{|r|}{ RMR System } & \multicolumn{3}{|c|}{ Q System } \\
\hline & \multirow{2}{*}{$\begin{array}{l}\text { Rock } \\
\text { class }\end{array}$} & \multirow{2}{*}{ Estimated Support } & \multirow{2}{*}{ Rock class } & \multicolumn{2}{|c|}{ Estimated Support } \\
\hline & & & & Shotcrete (mm) & Bolts \\
\hline $0+876-1+000$ & & & Fair rock & No & $\begin{array}{l}\text { Systematic bolting, with } 2 \mathrm{~m} \\
\text { length and } 1.8 \mathrm{~m} \text { spacing }\end{array}$ \\
\hline $1+000-2+800$ & & & & & \\
\hline $2+800-4+000$ & & $\begin{array}{l}\text { Systematic bolts of } 4 \mathrm{~m} \text { long, } \\
\text { spaced } 1.5-2 \mathrm{~m} \text { in crown }\end{array}$ & & $40-100$ & $\begin{array}{l}\text { Systematic bolting, with } 2 \mathrm{~m} \\
\text { length and } 2 \mathrm{~m} \text { spacing }\end{array}$ \\
\hline $4+000-5+600$ & Fair & $\begin{array}{l}\text { and walls with wire mesh in crown } \\
\text { and shotcrete of } 50-100 \mathrm{~mm} \\
\text { in crown and } 30 \mathrm{~mm} \text { in walls }\end{array}$ & Poor rock & No & $\begin{array}{l}\text { Systematic bolting, with } 2 \mathrm{~m} \\
\text { length and } 1.5 \mathrm{~m} \text { spacing }\end{array}$ \\
\hline $5+600-6+600$ & & & & $40-100$ & $\begin{array}{l}\text { Systematic bolting, with } 2 \mathrm{~m} \\
\text { length and } 2 \mathrm{~m} \text { spacing }\end{array}$ \\
\hline $6+600-7+000$ & & & & No & $\begin{array}{c}\text { Systematic bolting, with } 2 \mathrm{~m} \\
\text { length and } 1.6 \mathrm{~m} \text { spacing }\end{array}$ \\
\hline $7+200-7+506$ & Poor & $\begin{array}{c}\text { Systematic bolts of } 4-5 \mathrm{~m} \text { long, spaced } \\
1-1.5 \mathrm{~m} \text { in crown and walls with wire } \\
\text { mesh, shotcrete: } 100-150 \mathrm{~mm} \text { in } \\
\text { crown and } 100 \mathrm{~mm} \text { in walls with steel } \\
\text { sets of Light to medium ribs spaced } 1.5 \\
\text { m where required }\end{array}$ & $\begin{array}{l}\text { Very poor } \\
\text { rock }\end{array}$ & $\begin{array}{l}\text { Fiber reinforced } \\
(50-90 \mathrm{~mm})\end{array}$ & $\begin{array}{l}\text { Systematic bolting, with } 2 \mathrm{~m} \\
\text { length and } 1.5 \mathrm{~m} \text { spacing }\end{array}$ \\
\hline
\end{tabular}

Table 11. The optimized support for tunnel by using numerical models of $\mathrm{RS}^{3}$.

\begin{tabular}{|c|c|c|c|c|c|c|c|}
\hline \multirow{2}{*}{ Chainage } & \multirow{2}{*}{$\begin{array}{c}\text { Shotcrete } \\
(\mathrm{mm})\end{array}$} & \multicolumn{2}{|c|}{ Systematic bolts } & \multirow{2}{*}{ Chainage } & \multirow{2}{*}{$\begin{array}{c}\text { Shotcrete } \\
(\mathrm{mm})\end{array}$} & \multicolumn{2}{|c|}{ Systematic bolts } \\
\hline & & Length (m) & Spacing (m) & & & Length (m) & Spacing (m) \\
\hline
\end{tabular}




\section{Continued}

\begin{tabular}{|c|c|c|c|c|c|c|c|}
\hline $0+876-1+000$ & 50 & 2 & 1.8 & $4+400-4+800$ & 40 & 2 & 1.5 \\
\hline $1+000-1+600$ & 50 & 2 & 2 & $4+800-5+200$ & 40 & 2 & 1.5 \\
\hline $1+600-2+000$ & 50 & 2 & 2 & $5+200-5+600$ & 40 & 2 & 1.5 \\
\hline $2+000-2+400$ & 50 & 2 & 2 & $5+600-6+000$ & 50 & 2 & 2 \\
\hline $2+400-2+800$ & 80 & 2 & 2 & $6+000-6+600$ & 50 & 2 & 2 \\
\hline $2+800-3+200$ & 50 & 2 & 2 & $6+600-7+000$ & 30 & 2 & 1.5 \\
\hline $3+200-3+600$ & 50 & 2 & 2 & $7+000-7+200$ & 80 & 3 & 1.5 \\
\hline $3+600-4+000$ & 50 & 2 & 2 & & & & \\
\hline $4+000-4+400$ & 40 & 2 & 1.5 & $7+200-7+506$ & 100 & 2 & 1.5 \\
\hline
\end{tabular}
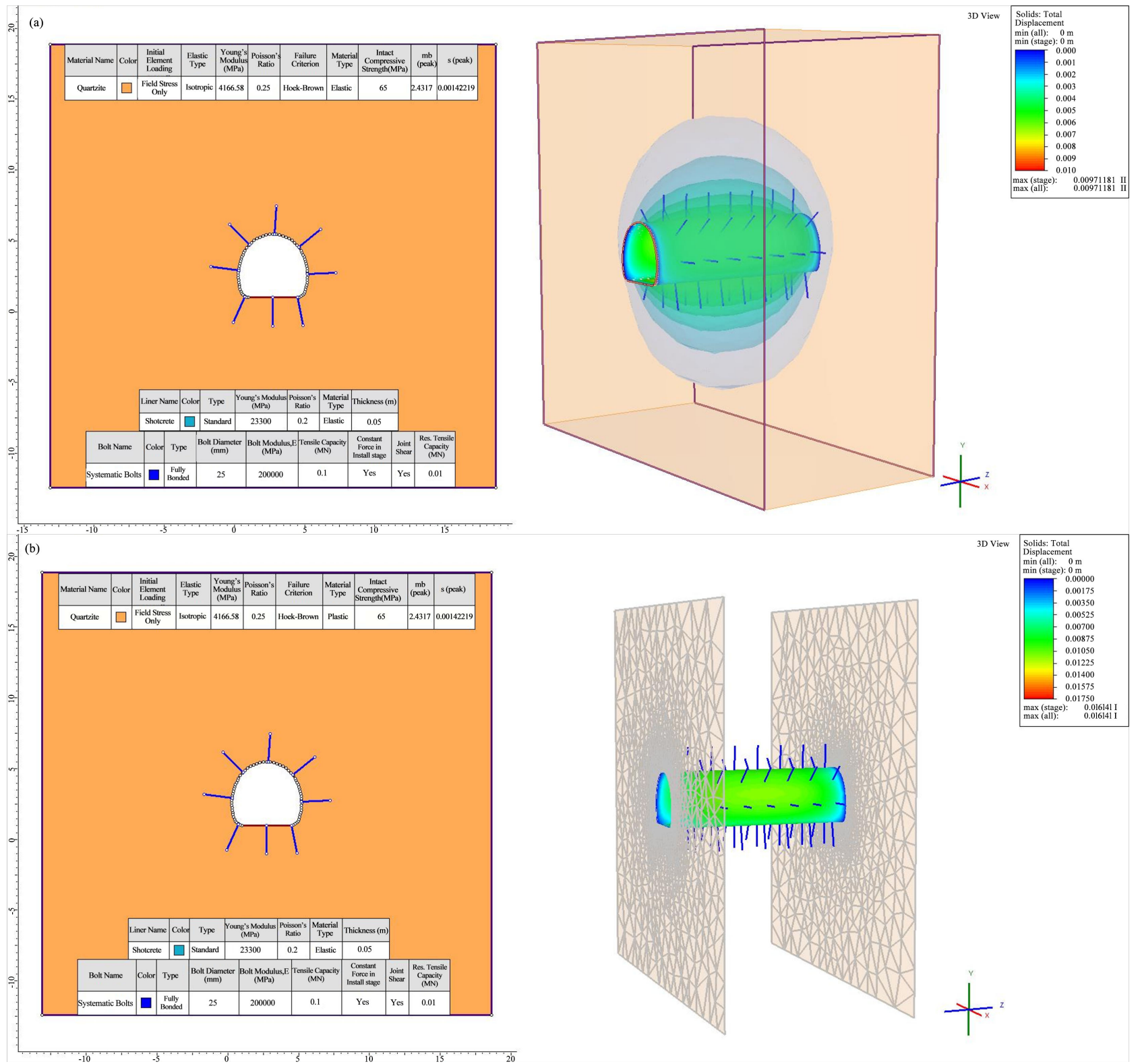


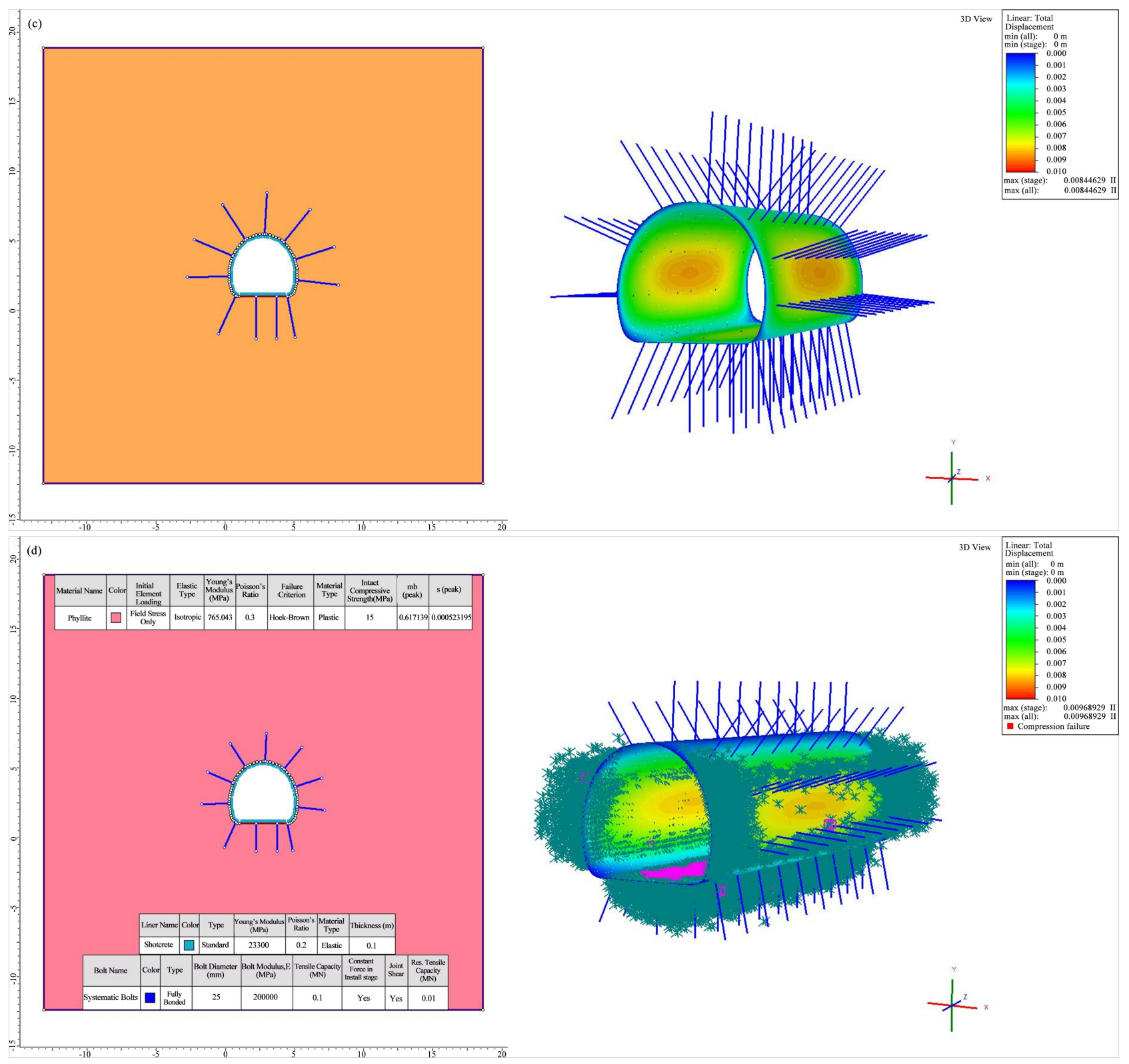

Figure 7. (a) Displacement contours support in elastic conditions along 1+000 - 1+600 section; (b) 3D view showing liner's displacement with deformation mesh along boundary along 2+400 - 2+800; (c) Showing supported total displacement in plastic conditions along section 7+000 - 7+200; (d) Yielded element along excavation in Plastic conditions along chainage 7+200 - 7+506.

\section{Conclusion}

In this study classification of the rock mass, prediction of rock burst, squeezing and deformation modulus of rock mass were assessed along headrace tunnel of hydropower which is $7.506 \mathrm{~km}$ long and $5 \mathrm{~m}$ in diameter. Initially, rock mass was classified by using three empirical methods i.e. RMR, Q, RMi and it is concluded by comparing the results of these methods that rock mass from chainage $0+876$ to $7+000$ lies in fair rock and from 7+000 to 7+506 lies in poor rock class. Empirical relations proposed by several researchers were used to determine the rock burst and squeezing potential of the rock mass and comparison of results 
depicts that medium stress conditions exist at $1+000$ to $4+000$ which can induce rock spalling and rock burst. The chainage $2+400-2+800$ has maximum tunnel depth $(640 \mathrm{~m})$ which can cause brittle failures in rock mass and medium rock burst can also be observed. The remaining sections may face minor spalling and light bursting. Initially, rock bolts of avg. $2 \mathrm{~m}$ long with shotcrete of $30-100 \mathrm{~mm}$ in thickness were estimated by RMR and $\mathrm{Q}$ to use in numerical analyses. In section $7+000$ to $7+200$ length of the bolts are increased to $3 \mathrm{~m}$ due to widely spaced joints with a high aperture. The total displacement of both elastic and plastic condition was measured by analyzing the numerical models in both supported and unsupported conditions for every section along tunnel alignment. In future, the detail study will be required with the help of subsurface drilling data.

\section{Conflicts of Interest}

The authors declare no conflicts of interest regarding the publication of this paper.

\section{References}

[1] Panthi, K.K. (2012) A Probabilistic Approach in Assessing Tunnel Squeezing-A Discussion Based on Tunnel Projects from Nepal Himalaya. 46th US Rock Mechanics/Geo-Mechanics Symposium, Chicago, Illinois, 24-27 June 2012, 467-482.

[2] Yaxun, X., Feng, X.T. and Li, S. (2016) Rock Mass Failure Mechanisms during the Evolution Process of Rock Bursts in Tunnels. International Journal of Rock Mechanics and Mining Sciences, 83, 174-181.

https://doi.org/10.1016/j.ijrmms.2016.01.008

[3] Zhang, C., Feng, X.T. and Zhou, H. (2013) Rockmass Damage Development Following Two Extremely Intense Rock Bursts in Deep Tunnels at Jinping II Hydropower Station, Southwestern China. Bulletin of Engineering Geology and the Environment, 72, 237-247. https://doi.org/10.1007/s10064-013-0470-y

[4] Gong, Q.M., Yin, L.J. and She, Q.R. (2013) TBM Tunneling in Marble Rock Masses with High in Situ Stress and Large Groundwater Inflow: A Case Study in China. Bulletin of Engineering Geology and the Environment, 72, 163-172. https://doi.org/10.1007/s10064-013-0460-0

[5] Kaya, A., Karaman, K. and Bulut, F. (2017) Geotechnical Investigations and Remediation Design for Failure of Tunnel Portal Section: A Case Study in Northern Turkey. Journal of Mountain Science, 14, 1140-1160. https://doi.org/10.1007/s11629-016-4267-x

[6] Ritter, W. (1879) Die Statik der Tunnelgewölbe. Springer, German.

[7] Naithani, A.K., Bhatt, A.K. and Murthy, K.S.K. (2009) Geological and Geotechnical Investigations of Loharinag-Pala Hydroelectric Project, Garhwal Himalaya, Uttarakhand. Journal of the Geological Society of India, 73, 821-836. https://doi.org/10.1007/s12594-009-0066-0

[8] Gupta, M.C., Singh, B.K. and Singh, K.N. (2011) Engineering Geological Rock Mass Classification of Punasa Tunnel Site, Khandwa District, Madhya Pradesh. Journal of the Geological Society of India, 77, 269-272.

https://doi.org/10.1007/s12594-011-0034-3

[9] Panthee, S., Singh, P.K., Kainthola, A., et al. (2018) Comparative Study of the De- 
formation Modulus of Rock Mass. Bulletin of Engineering Geology and the Environment, 77, 751-760.

[10] Palmstrom, A. and Singh, R. (2001) The Deformation Modulus of Rock Masses: Comparisons between in Situ Tests and Indirect Estimates. Tunnelling and Underground Space Technology, 16, 115-131. https://doi.org/10.1016/S0886-7798(01)00038-4

[11] Hoek, E. and Diederichs, M.S. (2006) Empirical Estimation of Rock Mass Modulus. International Journal of Rock Mechanics and Mining Sciences, 43, 203-215. https://doi.org/10.1016/j.ijrmms.2005.06.005

[12] Shen, J., Karakus, M. and Xu, C. (2012) A Comparative Study for Empirical Equations in Estimating Deformation Modulus of Rock Masses. Tunnelling and Underground Space Technology, 32, 245-250. https://doi.org/10.1016/j.tust.2012.07.004

[13] Ajalloeian, R. and Mohammadi, M. (2014) Estimation of Limestone Rock Mass Deformation Modulus Using Empirical Equations. Bulletin of Engineering Geology and the Environment, 73, 541-550. https://doi.org/10.1007/s10064-013-0530-3

[14] Karaman, K., Ferdi, C. and Ayhan, K. (2015) A Comparative Assessment of Rock Mass Deformation Modulus. International Journal of Mining Science and Technology, 25, 735-740. https://doi.org/10.1016/j.ijmst.2015.07.006

[15] Bieniawski, Z.T. (1978) Determining Rock Mass Deformability: Experience from Case Histories. International Journal of Rock Mechanics and Mining Sciences, 15, 237-247. https://doi.org/10.1016/0148-9062(78)90956-7

[16] Gardner, W.S. (1987) Design of Drilled Piers in the Atlantic Piedmont. In: Smith, R.E., Ed., Foundations and Excavations in Decomposed Rock of the Piedmont Province, Vol. 9, ASCE, Reston, 62-86.

[17] Palmstrom, A. (1996) RMi-A Rock Mass Characterization System for Rock Engineering Purposes. PhD Thesis, The University of Oslo, Norway.

[18] Barton, N. (2002) Some New Q-Value Correlations to Assist in Site Characterization and Tunnel Design. International Journal of Rock Mechanics and Mining Sciences, 39, 185-216. https://doi.org/10.1016/S1365-1609(02)00011-4

[19] Kayabasi, A., Gokceoglu, C. and Ercanoglu, M. (2003) Estimating the Deformation Modulus of Rock Masses: A Comparative Study. International Journal of Rock Mechanics and Mining Sciences, 40, 55-63. https://doi.org/10.1016/S1365-1609(02)00112-0

[20] Zhang, L. and Einstein, H.H. (2004) Using RQD to Estimate the Deformation Modulus of Rock Masses. International Journal of Rock Mechanics and Mining Sciences, 41, 337-341. https://doi.org/10.1016/S1365-1609(03)00100-X

[21] Gurocak, Z., Solanki, P. and Zaman, M.M. (2007) Empirical and Numerical Analyses of Support Requirements for a Diversion Tunnel at the Boztepe Dam Site, Eastern Turkey. Engineering Geology, 91, 194-208. https://doi.org/10.1016/j.enggeo.2007.01.010

[22] Serafim, J.L. and Pereira, J.P. (1983) Considerations on the Geomechanical Classification of Bieniawski. Proceedings of International Symposium on Engineering Geology and Underground Openings, Lisbon, Portugal, 1983, 1133-1144.

[23] Nicholson, G.A. and Bieniawski, Z.T. (1990) A Nonlinear Deformation Modulus Based on Rock Mass Classification. International Journal of Mining and Geological Engineering, 8, 181-202. https://doi.org/10.1007/BF01554041

[24] Mitri, H.S., Edrissi, R. and Henning, J. (1994) Finite Element Modeling of Cable Bolted Stopes in Hard Rock Ground Mines. Proceedings of SME Annual Confe- 
rence, Albuquerque, NM, USA, 14-17 February 1994, 94-116.

[25] Gokceoglua, C., Sonmeza, H. and Kayabasi, A. (2003) Predicting the Deformation Moduli of Rock Masses. International Journal of Rock Mechanics and Mining Sciences, 40, 701-710. https://doi.org/10.1016/S1365-1609(03)00062-5

[26] Gurocak, Z. (2011) Analyses of Stability and Support Design for a Diversion Tunnel at the Kapikaya Dam Site, Turkey. Bulletin of Engineering Geology and the Environment, 70, 41-52. https://doi.org/10.1007/s10064-009-0258-2

[27] Hamid, R.N., Abdolhadi, G. and Seyed, A.M. (2014) On the Use of the RMR System for Estimation of Rock Mass Deformation Modulus. Bulletin of Engineering Geology and the Environment, 73, 531-540. https://doi.org/10.1007/s10064-013-0522-3

[28] Rocscience Inc. (2014) $\mathrm{RS}^{3}$ Version 1.0-Finite Element Analysis for Excavations and Slopes. Toronto, ON.

[29] Rocscience Inc. (2014) Dips Version 7.0-Graphical and Statistical Analysis of Orientation Data. Toronto, ON. http://www.rocscience.com

[30] Palmstrom, A. (2005) Measurements of and Correlations between Block Size and Rock Quality Designation (RQD). Tunnelling and Underground Space Technology, 20, 362-377. https://doi.org/10.1016/j.tust.2005.01.005

[31] Palmstrom, A. (1982) The Volumetric Joint Count a Useful and Simple Measure of the Degree of Jointing. Proceedings of 4 th International Congress IAEG, New Delhi, 10-15 December 1982, 221-228.

[32] Palmstrom, A. (1985) Application of the Volumetric Joint Count as a Measure of Rock Mass Jointing. Proceedings of International Symposium on Fundamentals of Rock Joints, Björkliden, 15-20 September 1985, 103-111.

[33] Palmstrom, A. (1986) A General Practical Method for Identification of Rock Masses to Be Applied in Evaluation of Rock Mass Stability Conditions and TBM Boring Progress. Proceedings of the Conference on Fjellsprengningsteknikk, Bergmekanikk, Geoteknikk, Oslo, Norway, 1986, 31.1-31.31.

[34] Sen, Z. and Eissa, E.A. (1992) Rock Quality Charts for Log-Normally Distributed Block Sizes. International Journal of Rock Mechanics and Mining Sciences \& Geomechanics Abstracts, 29, 1-12. https://doi.org/10.1016/0148-9062(92)91040-C

[35] Bieniawski, Z.T. (1973) Engineering Classification of Jointed Rock Masses. South African Institution of Civil Engineers, 15, 335-344.

[36] Bieniawski, Z.T. (1989) Engineering Rock Mass Classifications. Wiley, New York. $251 \mathrm{p}$.

[37] Barton, N.R., Lien, R. and Lunde, J. (1974) Engineering Classification of Rock Masses for the Design of Tunnel Support. Rock Mechanics, 4, 189-239. https://doi.org/10.1007/BF01239496

[38] Palmstrom, A. (1995) Characterizing the Strength of Rock Masses for Use in Design of Underground Structures. International Conference of Design and Construction of Underground Structures, New Delhi, 23-25 February 1995, 10 p.

[39] Singh, B., Jethwa, J.L. and Dube, A.K. (1992) Correlation between Observed Support Pressure and Rock Mass Quality. Tunnelling and Underground Space Technology, 7, 59-74. https://doi.org/10.1016/0886-7798(92)90114-W

[40] Goel, R.K., Jethwa, J.L. and Paithankar, A.G. (1995) Indian Experiences with Q and RMR Systems. Tunnelling and Underground Space Technology, 10, 97-109. https://doi.org/10.1016/0886-7798(94)00069-W

[41] Sengupta, S. (1998) Influence of Geological Structures on in Situ Stresses. PhD Thesis, Department of Civil Engineering, IIT, Uttarakhand, India, 275. 
[42] Hoek, E. and Brown, E.T. (1980) Underground Excavations in Rock. Institution of Mining and Metallurgy, London, 527.

[43] Russenes, B.F. (1974) Analysis of Rock Spalling for Tunnels in Steep Valley Sides (in Norwegian). Master's Thesis, Norwegian Institute of Technology, Department of Geology, Norway, 247.

[44] Grimstad, E. and Barton, N. (1993) Updating the Q-System for NMT, In: Proceedings of the International Symposium on Sprayed Concrete, Norwegian Concrete Association, Oslo, Norway, 20.

[45] Palmstrom, A. (1995) RMi-A for Rock Mass Characterization System for Rock Engineering Purposes. PhD Thesis, The University of Oslo, Norway, 400.

[46] ASTM (1995) Standard Test Method for Determination of the Point Load Strength Index of Rock (Withdrawn 2016). ASTM D 5731-95, West Conshohocken, PA.

[47] Read, S.A.L., Richards, L.R. and Perrin, N.D. (1999) Applicability of the Hoek-Brown Failure Criterion to New Zealand Greywacke Rocks. Proceedings of the 9 th International Congress on Rock Mechanics, Paris, France, 25-28 August 1999, 655-660.

[48] Eberhardt, E. (2001) Numerical Modelling of Three-Dimension Stress Rotation Ahead of an Advancing Tunnel Face. International Journal of Rock Mechanics and Mining Sciences, 38, 499-518. https://doi.org/10.1016/S1365-1609(01)00017-X

[49] Jing, L. and Hudson, J.A. (2002) Numerical Methods in Rock Mechanics. International Journal of Rock Mechanics and Mining Sciences, 39, 409-427.

https://doi.org/10.1016/S1365-1609(02)00065-5

[50] Vermeer, P.A., Moller, S.C. and Ruse, N. (2003) On the Application of Numerical Analysis in Tunnelling. Proceedings of the 12 th Asian Regional Conference on Soil Mechanics and Geotechnical Engineering, World Scientific, Singapore, 1-6.

[51] Lee, J.S. (2009) An Application of Three-Dimensional Analysis around a Tunnel Portal under Construction. Tunnelling and Underground Space Technology, 24, 731-738. https://doi.org/10.1016/j.tust.2009.06.003

[52] Verma, A.K. and Singh, T.N. (2010) Assessment of Tunnel Instability-A Numerical Approach. Arabian Journal of Geosciences, 3, 181-192.

https://doi.org/10.1007/s12517-009-0066-9

[53] Verma, A.K., Bajpai, R.K. and Singh, T.N. (2011) 3D Instability Analysis of an Underground Geological Repositorydan Indian Case Study. Arabian Journal of Geosciences, 4, 1173-1188. https://doi.org/10.1007/s12517-010-0131-4

[54] Kainthola, A., Singh, P.K., Wasnik, A.B., Sazid, M. and Singh, T.N. (2012) Finite Element Analysis of Road Cut Slopes Using Hoek \& Brown Failure Criterion. International Journal of Earth Science and Engineering, 5, 1100-1109.

[55] Singh, P.K., Wasnik, A.B. and Kainthola, A. (2013) The Stability of Road Cut Cliff Face along SH-121: A Case Study. Natural Hazards, 68, 497-507. https://doi.org/10.1007/s11069-013-0627-9

[56] Qiu, Y., Yang, X., You, C. and Xu, Q. (2013) Numerical Simulation Test of Tunnel's Deformation under Different Levels of Horizontal Stress. In: Fourth International Conference on Transportation Engineering, American Society of Civil Engineers, Chengdu, China, 2071-2075. https://doi.org/10.1061/9780784413159.301 